\title{
Multiclient Identification System Using Adaptive Probabilistic Model
}

\author{
Chin-Teng Lin, ${ }^{1}$ Linda Siana, ${ }^{1}$ Yu-Wen Shou, ${ }^{2}$ and Chien-Ting Yang ${ }^{1}$ \\ ${ }^{1}$ Department of Electrical and Control Engineering, National Chiao Tung University, Hsinchu 300, Taiwan \\ ${ }^{2}$ Department of Computer and Communication Engineering, China University of Technology, Hsinchu 303, Taiwan
}

Correspondence should be addressed to Yu-Wen Shou, owen@cute.edu.tw

Received 1 December 2009; Revised 26 February 2010; Accepted 14 April 2010

Academic Editor: Yingzi Du

Copyright (C) 2010 Chin-Teng Lin et al. This is an open access article distributed under the Creative Commons Attribution License, which permits unrestricted use, distribution, and reproduction in any medium, provided the original work is properly cited.

\begin{abstract}
This paper aims at integrating detection and identification of human faces in a more practical and real-time face recognition system. The proposed face detection system is based on the cascade Adaboost method to improve the precision and robustness toward unstable surrounding lightings. Our Adaboost method innovates to adjust the environmental lighting conditions by histogram lighting normalization and to accurately locate the face regions by a region-based-clustering process as well. We also address on the problem of multi-scale faces in this paper by using 12 different scales of searching windows and 5 different orientations for each client in pursuit of the multi-view independent face identification. There are majorly two methodological parts in our face identification system, including PCA (principal component analysis) facial feature extraction and adaptive probabilistic model (APM). The structure of our implemented APM with a weighted combination of simple probabilistic functions constructs the likelihood functions by the probabilistic constraint in the similarity measures. In addition, our proposed method can online add a new client and update the information of registered clients due to the constructed APM. The experimental results eventually show the superior performance of our proposed system for both offline and real-time online testing.
\end{abstract}

\section{Introduction}

Biometrics has been an emerging technology for identifying people by their physical and behavioral characteristics $[1,2]$, and its applications have attracted more and more attentions of researchers recently. Some physical characteristics of an individual could be used in biometric identification/verification system, such as fingerprint, palm print, face, and ear. Similarly, the behavioral characteristics included signature, speech, gesture, and gait. Among all biometric identification fields, face recognition has always been considered much more popular and significant. Face detection and recognition were also used in video surveillance and human computer interface. Furthermore, face recognition with the passive and nonintrusive benefits would be more appropriate for personal identifications.

A typical face recognition system was composed of two parts, face detection and face identification. It would be quite challenging for face detection to localize the faces in an image because the detected results might highly depend on the surrounding conditions such as environments, movements, lighting, orientations, and even the expressions of faces. These variant factors may lead to the changes of colors, luminance, shadows, and contours of images. For this reason, it is impractical to detect faces by using a single feature. Papageorgiou et al. [3] proposed a 2-D Haar feature to detect objects by using SVM (Support Vector Machine) in the training multiple Haar features. Li et al. [4] proposed a "Floatboost" algorithm to delete the worse face features to improve the detection rate and speed. Liehhart and Maydt [5] proposed the extensive set of Haar-like features for the rapid object detection, which gave the versatile uses of Haar features and improved the precisions of object detection. Then Viola and Jones [6] proposed three important methods to detect objects efficiently. First, they applied integral images to reducing the computational loadings of features. Second, 


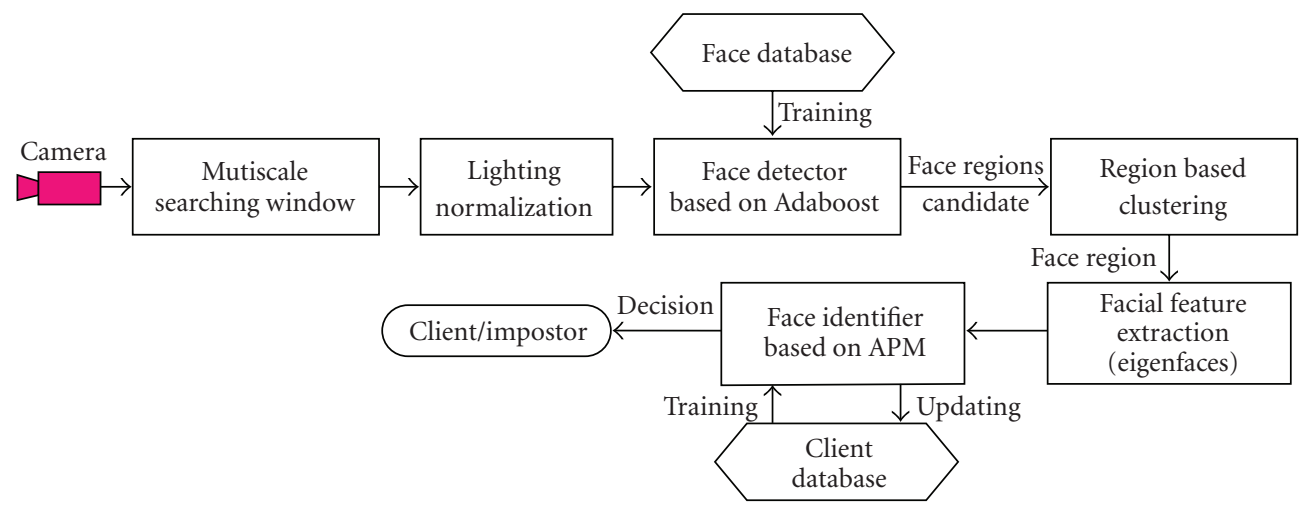

Figure 1: Architecture of the proposed face recognition system.

a simple and efficient classifier based on the Adaboost learning algorithm [7] was used to select a small number of features from a very large range of potential features. Third, they presented a cascaded-combined-classifier method to speed up the processing time.

Face identification was to identify faces in the registered database. While many approaches of face identification have aimed at identifying faces under slight changes of lighting, facial expressions, and poses, reliable techniques for identification under drastic variations have proven elusive. The major issue in the view-independent face identification was how to identify a registered face from different viewing directions. There were different kinds of methods for handling posed variations in face identification, including the invariant-feature method, 3D model-based method, and multiview method. The invariant-feature method attempted to extract features of faces from novel views, and uses these features to identify the faces [8-10]. One major disadvantage of this method was the unfeasibility of finding the sufficient invariant features for identification. The 3D model-based method focuses on constructing a prototypical view from a 3D model. As what [11] has mentioned, the 3D modelbased method can work well for faces with small angles of rotations. However, this kind of methods might fail for faces with larger rotations due to the invisibility of some important features [12]. The multiview method could be more significant since the sufficient number of faces in different views would be taken into consideration to deal with the pose problems [13]. Beymer [14] modeled faces from 15 views, and sampled different poses from the viewing sphere. The way of face identification consisted of two main stages, the geometrical alignment and correlation for matching. There have been also other works presented and proven to be robust to changes of viewpoints. One of them was the well-known single-view eigenspace approach, and its concept was based on the principal component analysis (PCA) [15-20]. Many related works were proposed to improve either the performance of face detection or face identification. However, a complete face recognition system including face detector and identifier was rarely proposed in the recent researches. Moreover, most of presented works were lack of flexibility to add new clients and to update the clients' information automatically. For more real-time applications, we in this paper tended to propose a practical system by integrating both face detection and identification systematically. Our proposed system used the cascaded Adaboost learning algorithm in face detection, and achieved the multiclient identification mechanism by using adaptive probabilistic model (APM). This paper would be organized in the following sections. First, our presented face detection system included histogram lighting normalization, feature selection, the cascaded Adaboost classifier, and the regionbased clustering algorithm. After that, the identification process including similarity measurement and the parameter adjustment by APM would be introduced. Finally, the experimental results and conclusions would be given to be summarized.

\section{Face Recognition System}

The architecture of face recognition system in our work was shown in Figure 1. The system consists of face detection system localizing the face regions in a captured image, and face identifier identifying "who" may belong to the extracted face. We presented a novel idea, the searching windows with various sizes, which would be used to find different face candidates in multiscales. The face candidates in different scales truly reflect various distances of clients from cameras. We totally define 12 searching windows in various sizes from the smallest block size of $24 \times 24$ to the biggest one by a multiplier of 1.25 . While a camera acquires an image, the camera would produce the images in different illuminating intensities depending on the light surrounding clients. Therefore, it would be necessary for an accurate recognition process to normalize the changes of light with respect to the surrounding environments.

2.1. Lighting Normalization. The lighting normalization is based on a histogram fitting method. The primary task of histogram fitting is to transform the candidate histogram $H(l)$ to the target one $G(l)$ for $l=0, \ldots, L-1$ where $L$ represents the number of discrete gray levels. Our target histogram $G(l)$ was chosen as the histogram of image closest to the mean of the face database. Both of the original $H(l)$ 


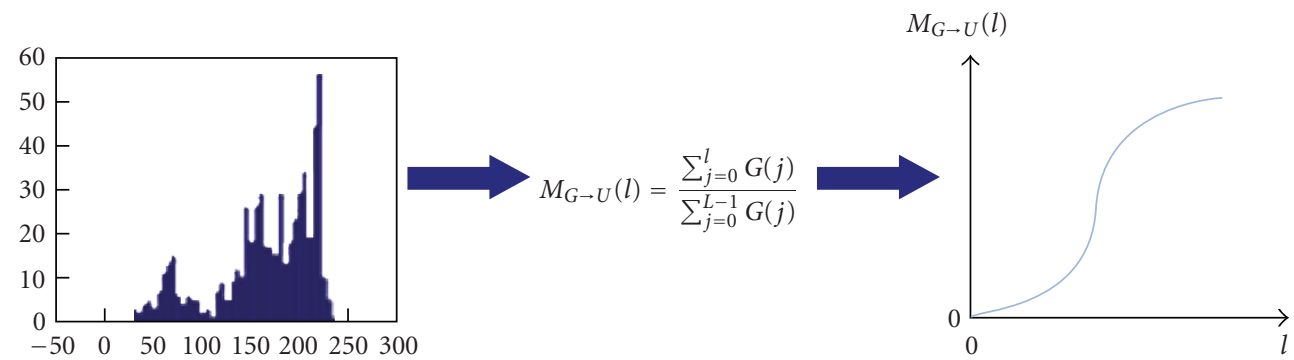

(a)
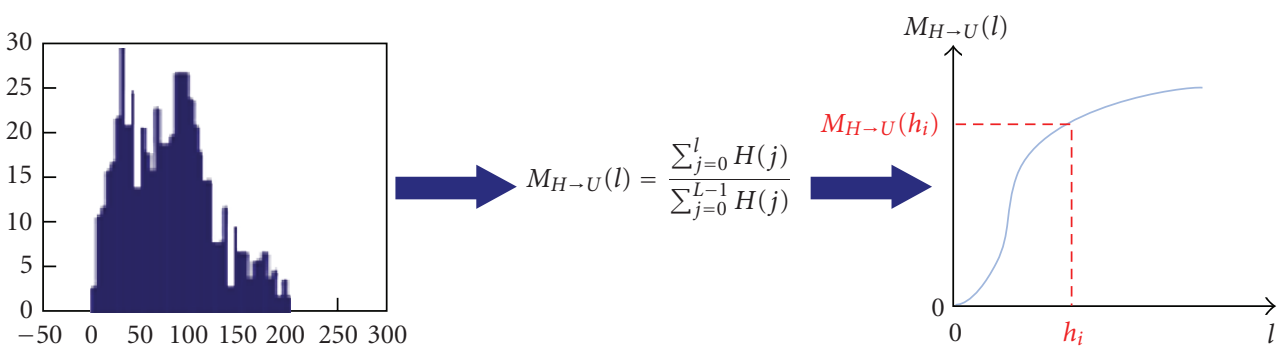

(b)

FIGURE 2: The candidate and target histograms, and their corresponding distributions.

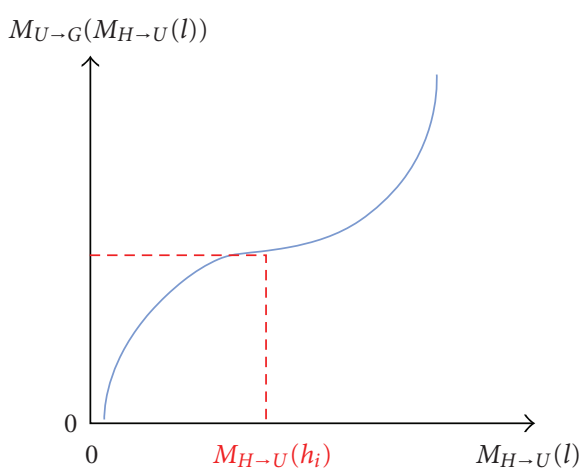

Figure 3: The distribution of $M_{H \rightarrow G}(l)$.

and target histogram $G(l)$ would be mapped to the uniform distributions $M_{H \rightarrow U}(l)$ and $M_{G \rightarrow U}(l)$

$$
M_{H \rightarrow U}(l)=\frac{\sum_{j=0}^{l} H(j)}{\sum_{j=0}^{L-1} H(j)}, \quad M_{G \rightarrow U}(l)=\frac{\sum_{j=0}^{l} G(j)}{\sum_{j=0}^{L-1} G(j)},
$$

where $M_{H \rightarrow U}(l)$ and $M_{G \rightarrow U}(l)$ are monotonically increasing. The histograms $H(l)$ can be mapped to $G(l)$ by $M_{H \rightarrow G}(l)$ in the following equation:

$$
M_{H \rightarrow G}(l)=M_{U \rightarrow G}\left(M_{H \rightarrow U}(l)\right) .
$$

$M_{U \rightarrow G}(l)$ denotes the inverse mapping of $M_{G \rightarrow U}(l)$. For each pixel in the original image, if the value of some pixel is $h_{i}$, we will firstly map $h_{i}$ to its corresponding value $M_{H \rightarrow U}(l)$ as shown in Figure 2. After that, $M_{H \rightarrow U}(l)$ will be mapped to $M_{H \rightarrow G}(l)$ by using the iterative scheme, which can be also illustrated in Figure 3. To demonstrate the practical changes after the lighting normalization, we showed the chosen target image $G(l)$ and the images before and after normalization in Figures 4(a), 4(b), and 4(c), respectively. The images with over-dark or over-light intensities would be normalized to the target one. Therefore, the histograms after lighting normalization would be similar to the histograms of targets.

2.2. Feature Selection. The intensity based features employed in this paper were based on Haar features. We selected four types of rectangle features as illustrated in Figure 5, including the vertical edge, horizontal edge, vertical line, and diagonal edge proposed by Papageorgiou [3]. In fact, it is feasible to use the composition of different brightness rectangles to represent the light and dark regions in the image. The features are defined in the following equation:

$$
\text { valve }_{\text {subtracted }}=f(x, y, w, h, \text { Type }),
$$

where $(x, y)$ indicate the origin of the relative coordinate of rectangle features in the searching window. The significance of $w$ and $h$ denote the relative weight and height of rectangle features, respectively. Type presents the type of rectangle

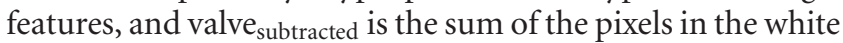
rectangle subtracted from those in the black ones.

A single rectangle feature which best separates the face and nonface samples can be considered as a weak classifier $h(x, f, p, \theta)$ as shown in the following equation:

$$
h(x, f, p, \theta)= \begin{cases}1, & \text { if } p f(x)<p \theta \\ 0, & \text { otherwise. }\end{cases}
$$

The weak classifier $h(x, f, p, \theta)$ used to determine if the $x$-block image is a face or a nonface depends on the feature $f(x, y, w$, and $h$ type $)$, the threshold $\theta$, and the polarity $p$ 


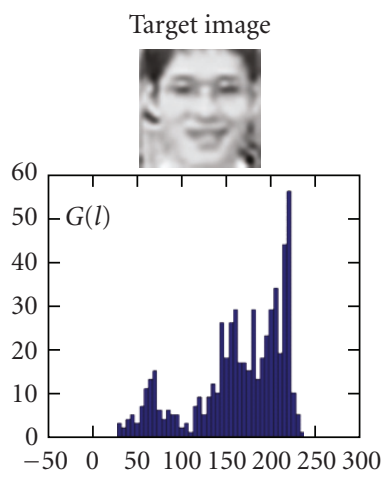

(a)
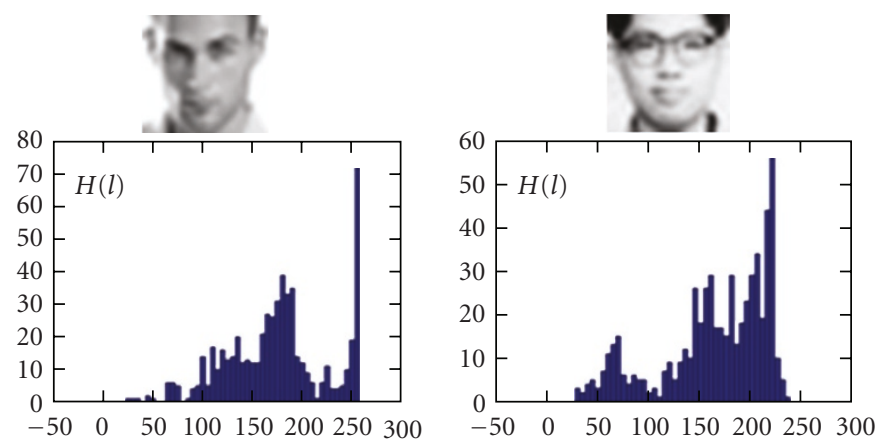

(b)

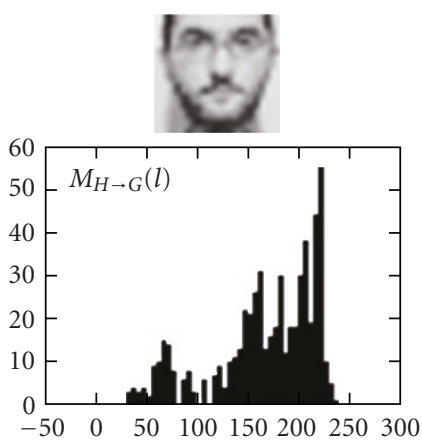

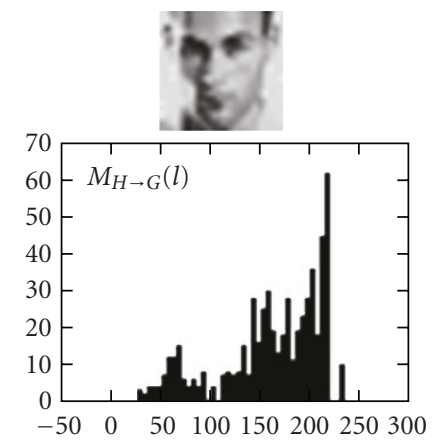

(c)

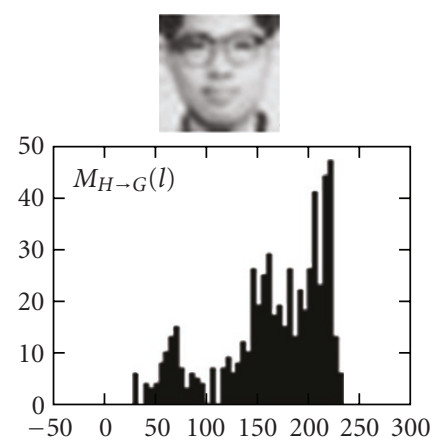

Figure 4: Lighting normalization. (a) Target image, (b) Input images, (c) Lighting normalized images.

$(x, y)$

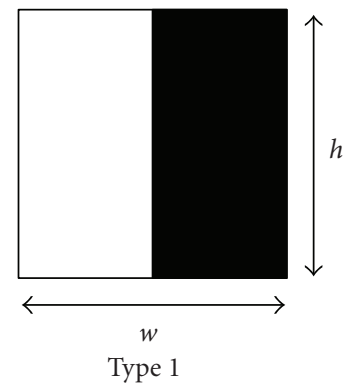

(a) $(x, y)$

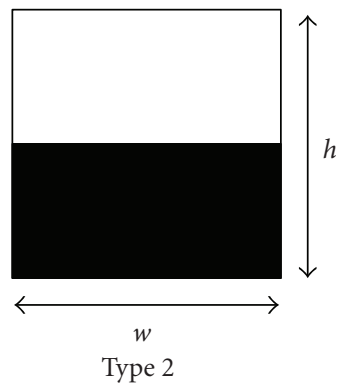

(b) $(x, y)$

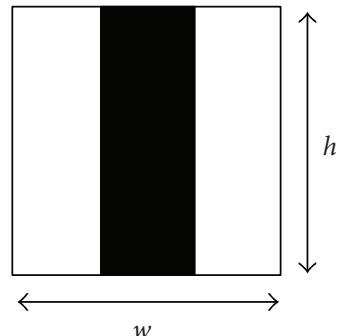

Type 3

(c) $(x, y)$

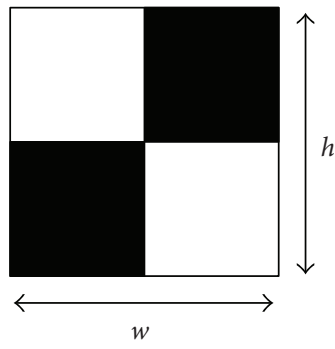

Type 4

(d)

FIGURe 5: Four types of rectangle features.

indicating the signs of inequality. For each weak classifier, an optimal threshold is chosen to minimize the possibilities of misclassifications. The selected threshold for each rectangle feature is acquired through the training process by our database which consists of 4000 face images and 59000 nonface images. Figures $6(\mathrm{a})$ and $6(\mathrm{~b})$ present some face and nonface examples in our database. In this procedure, we could collect the distributions of $f(x, y, w$, and $h$ type) for each image in the database, and then a threshold with higher distinguishability in clustering would be chosen. Although each rectangle feature can be obtained easily, computing the complete set of all features is extremely costing. Take the smallest searching window of $24 \times 24$ block size, for example, the entire number of rectangle features will be 160,000.

The Adaboost method combines a collection of weak classifiers to form a stronger classifier. Since the stronger classifier is rather time consuming, the structure of cascaded classifiers by Viola and Jones [6] will be preferred to improving the detection performance and reducing the computational time. As a result, our cascaded Adaboost classification based on the stronger classifier will classify each extracted face image step by step. In each step, only the image-block classified as a face may be essential to go to the next step. The number of steps must be sufficient to achieve 

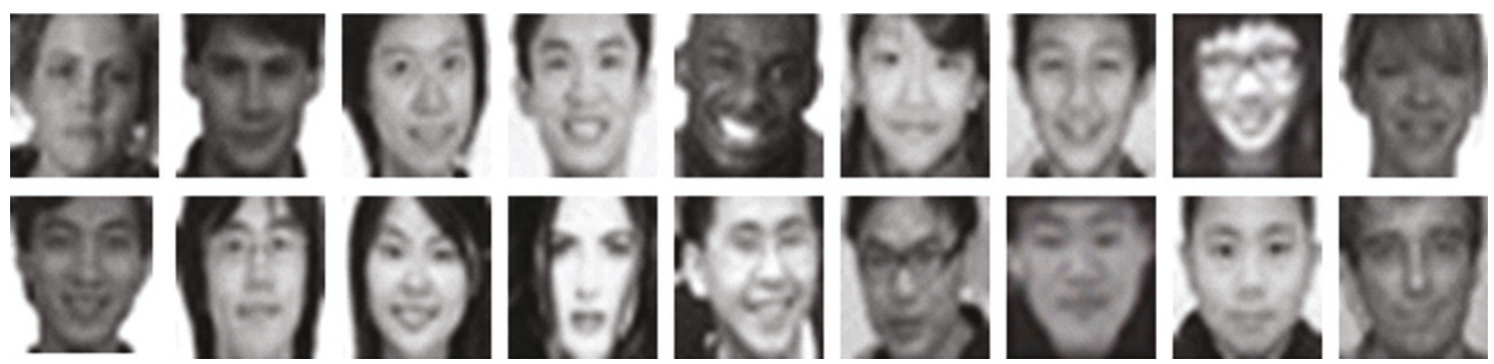

(a)
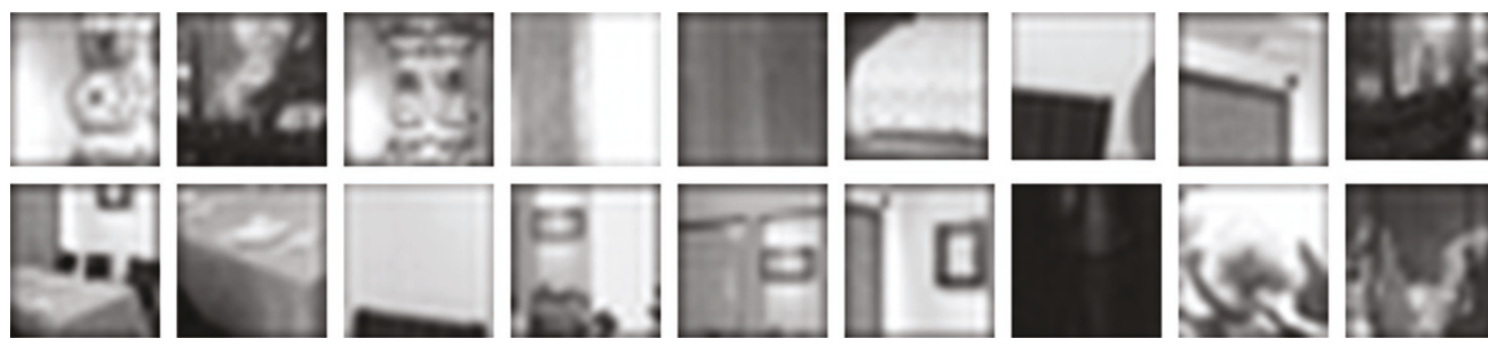

(b)

FIgURE 6: Database of face detection system. (a) Face images, (b) Non-face images.

an excellent detection rate and the minimized computational loading. For example, a detection rate of 0.9 can be achieved by 10-step classifier for the detection rate of 0.99 in each step $\left(0.9 \approx 0.99^{10}\right)$. The procedure of our implemented Adaboost process can be simply described as the following equations. If $m$ and $l$ are the number of nonface and face samples, respectively, and $j$ is the sum of nonface and face samples, the initial weight $w_{i, j}$ for the $i$ th-stage can be defined as $w_{i, j}=1 / 2 m,(1 / 2 l)$ for $y_{j}=0,1$. The normalized weighted error with respect to the weak classifier can be expressed in the following equation:

$$
\varepsilon_{i}=\min _{f, p, \theta} \sum_{j} w_{i, j}\left|h\left(x_{j}, f, p, \theta\right)-y_{j}\right| .
$$

The updating weights for each iteration are defined in (6) where $e_{j}$ equals to 0 if the object is classified correctly and vice versa:

$$
w_{i, j}=w_{i, j} \beta_{i}^{1-e_{j}}
$$

Also, the final classifier for the ith-stage is defined in the following equation:

$$
C\left(x_{j}\right)= \begin{cases}1, & \alpha_{i} h\left(x_{j}, f, p, \theta\right) \geq \frac{1}{2} \alpha_{i}, \\ 0, & \text { otherwise }\end{cases}
$$

where $\alpha_{i}=\log \left(1 / \beta_{i}\right)$ and $\beta_{i}=\varepsilon_{i} /\left(1-\varepsilon_{i}\right)$

2.3. Region Based Clustering. The face detector usually finds more than one face candidate even though only one single face appears in an image, which is illustrated in Figure 8, and a region-based clustering method is used to solve this kind of problems. The proposed region-based clustering method consists of two levels of clustering, local and global scale clustering. The local scale clustering is used to cluster the blocks in the same scale and design a simple filter to determine the number of blocks within clusters. While the number of blocks in some cluster is more than one, that cluster will be reserved as the possible candidate of faces, otherwise it will be discarded. The local scale clustering judges if the blocks meet the decision rule in (8). In (8), the overlap_rate $(x, y)$ is the percentage overlapped between two detected regions, $x$ and $y$, and distance $(x, y)$ is the distance of centers in these two regions. The equality,cluster $(x, y)=1$ means the block $x$ and $y$ are in the same cluster and the regions are completely overlapped

$$
\operatorname{cluster}(x, y)=\left\{\begin{aligned}
1, & \text { if overlap_rate }(x, y) \geq \mathrm{TH}_{\text {overlap_rate }} \\
& \text { and distance }(x, y) \leq \mathrm{TH}_{\text {distance }} \\
0, & \text { otherwise. }
\end{aligned}\right.
$$

Figure 9 shows several cases of the clustering process. In Figure 9(a), the two blocks are processed as the same cluster, and in Figure 9(b) the two blocks are processed as different clusters because the distance of the centers does not satisfy distance $(x, y) \leq \mathrm{TH}_{\text {distance. }}$. For the special case as shown in Figure 9(c), they are all considered as face candidates but most of them are false accept blocks. Therefore in this paper for practical applications, we only choose one block that satisfies overlap_rate $(x, y) \geq \mathrm{TH}_{\text {overlap_rate }}$ rather than select multiple blocks. At the end, the global scale clustering will use the blocks obtained from local scale clustering, and label the face regions by the average size of all available blocks. Some results in the entire region based clustering process for both local-scale and global-scale levels will be shown in Figure 10. From the right image in Figure 8, in fact, only 


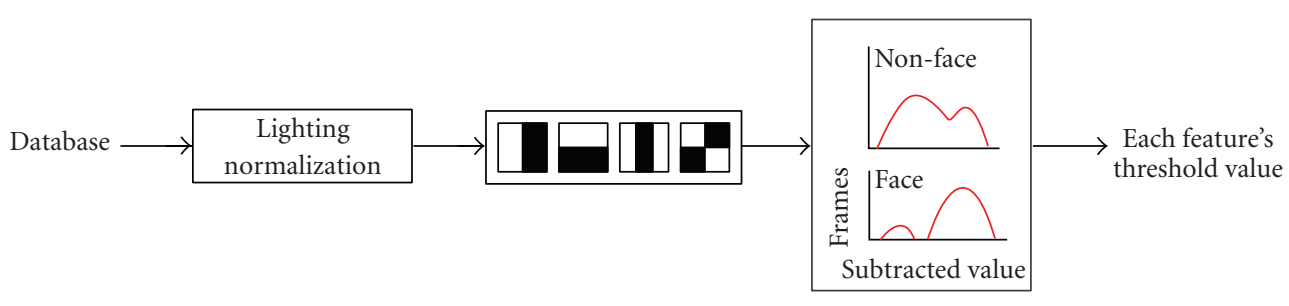

FIGURE 7: The process in selecting the threshold value for features in each rectangle.

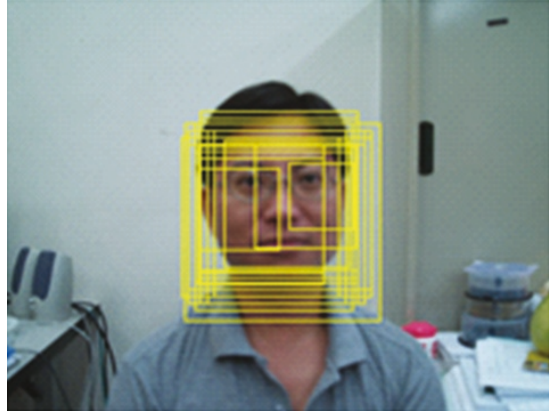

(a)

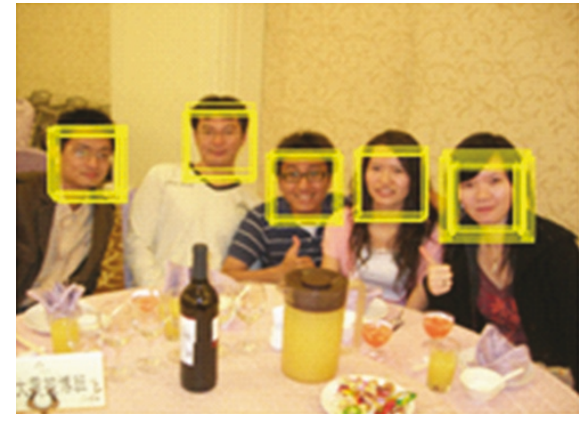

(b)

Figure 8: Some results in face detector.
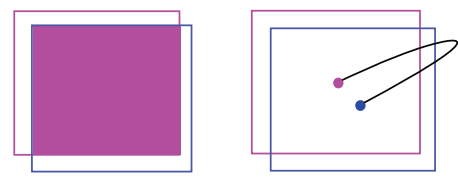

(a)

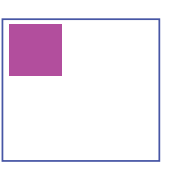

(b)

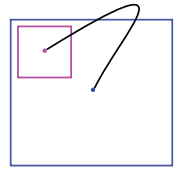

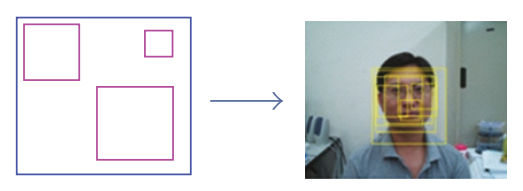

(c)

Figure 9: Examples of overlapped regions and the distance of centers of two blocks, (a) the same cluster, (b) two different clusters, (c) a special case, more than two blocks overlapping.

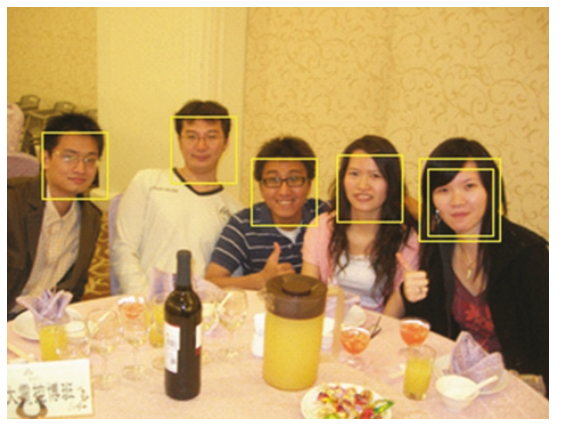

(a)

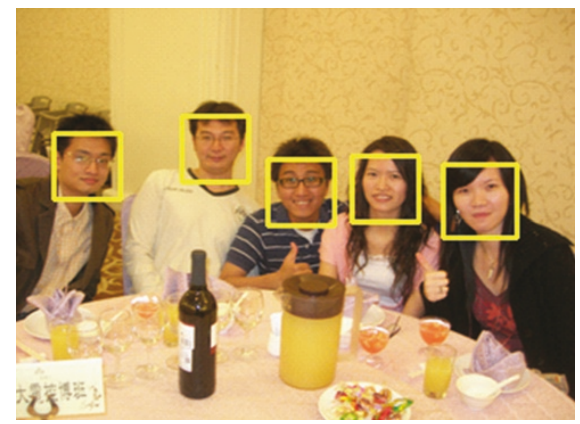

(b)

FIgURE 10: The results in the region based clustering, (a) for the local-scale clustering process and (b) for the global-scale clustering process. 


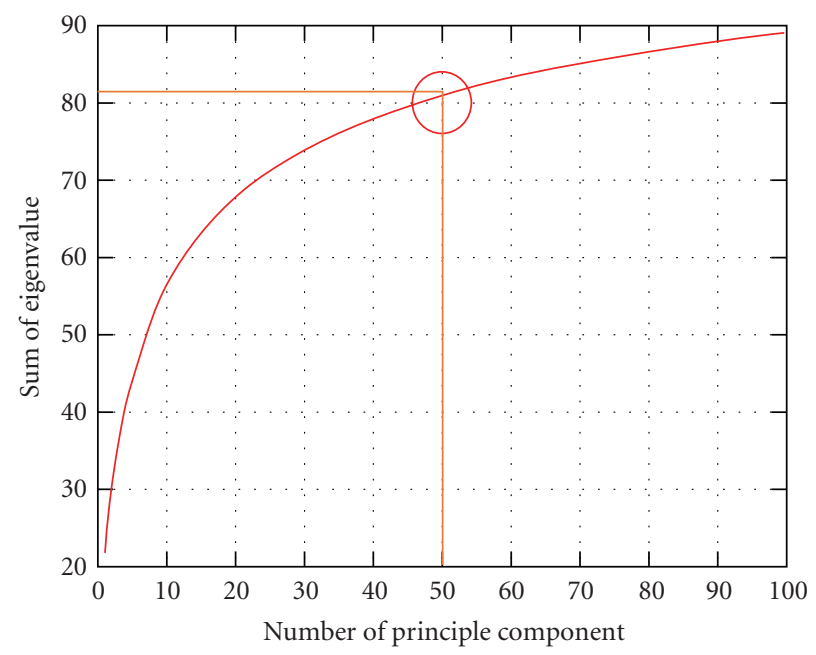

(a)

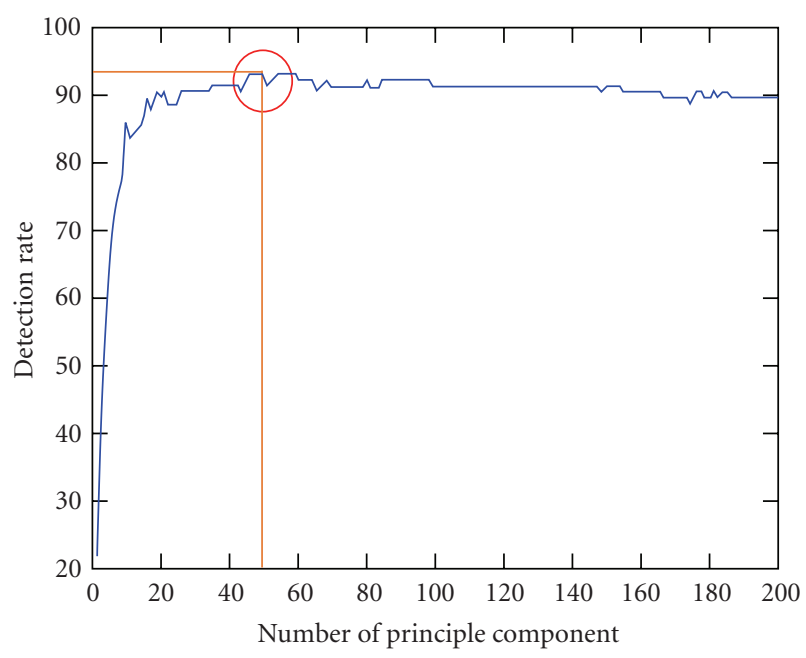

(b)

FIGURE 11: The pattern information for the specific dimensions of image subspace (a) the sum of eigenvalues and (b) the detection rate with respect to the number of eigenvectors.
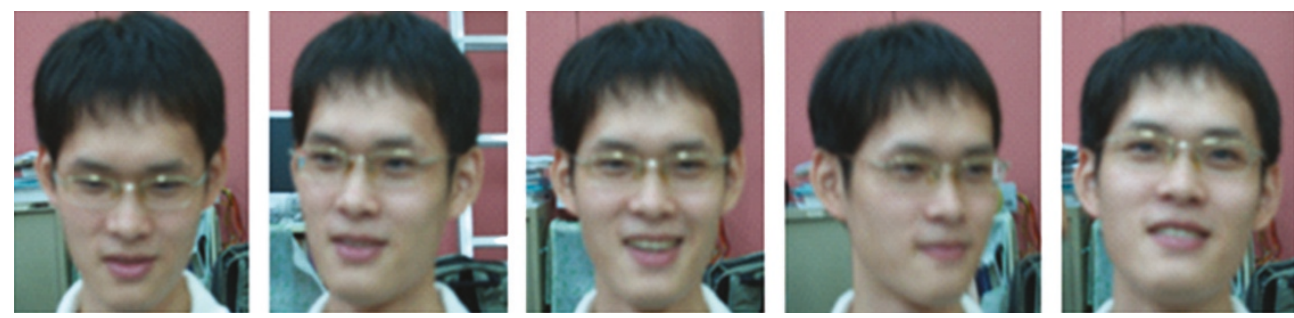

FIGURE 12: Five different head orientations of a client.

one block will be precisely clustered as a face region after applying our local and global clustering processes even though more than five face candidates are obtained for an image with only five faces.

\section{Face Identification}

We have two major parts of face identification in this work, facial feature extraction and adaptive probabilistic model (APM). The facial feature extractor is constructed by principle component analysis (PCA) [17] which effectively reduces the number of dimensions by maximizing the projections of scatters of all samples. To begin with, we have a training set of $N$ images and each image consists of $n$ elements. In our case, $N$ equals to 4000 which indicates the total number of images in the database. Each image has $n$ elements with the size equaling to $24 \times 24$ or a 576 -element vector.

The process of obtaining a single space consists of finding the covariance matrix $C$ of the training set and computing the eigenvectors $v_{k}$ for $k=1,2, \ldots, n$. The eigenvectors $v_{k}$ corresponding to the largest eigenvalues $\lambda_{k}$ span the base of searching subspaces. Each original image can be projected into the subspace as in the following equation:

$$
\eta_{k}=v_{k}^{T} \cdot \Phi_{s} \quad k=1,2, \ldots, m,
$$

where $m(m<n)$ is the chosen dimensionality of the image subspace, and $\Phi_{s}=\Gamma_{s}-\Psi$ represents the relation of $\Gamma_{s}$, a set of projected training images, and $\Psi$, the average image of the training set. If $m$ is closer to $n$, the results of face identification will be more precise. But the face identification takes more computational time to project the original images into the corresponding subspaces. Hence, we have to choose the appropriate dimensionality of image subspaces. Figure 11 shows one instance of how we determine the number of image subspaces. Figure 11(a) indicates that the pattern information about representative facial features will gain when the number of principle components increases. But the detection rate in Figure 11(b) will be saturated to a limit value or even reduced when the number of principle components is larger than the specific value (50 in this case). The reason is simple that the pattern information may include both the significant information and noise, which makes more eigenvectors extracted more 
noises involved. Therefore, how to determine the optimal number of eigenvectors will be much more significant, and this idea contributes to one of the major points in this paper. We can easily observe that the performance of the detection rate descends by the effect of noises. From the observations in simulations, we can determine the number of eigenvectors in our face identification system by the pattern information and detection rate in about $81 \%$ and $93 \%$, respectively.

3.1. Similarity Measure. The APM method is proposed to achieve the faster and functional goals for face identification. It can online register new clients and update the clients' information. This capability can enhance the practicability and heighten the identification rate of the proposed system for more other applications. The primary concept of APM architecture is based on the view-independent face identification. The view-independent model of face identification is more robust than the single-view one because the head orientations of a person may be changeable in the real conditions. In our proposed system, the view-independent model of face identification system is built with five different head orientations for each client as shown in Figure 12. The APM method follows the probabilistic constraint in the similarity measures to design a model of likelihood functions, since the judgment rules of classifications depend on the degree of likelihoods. We denote a testing sample $x$, and the similarity between $x$ and each registered client can be computed by the likelihood function of each client. The testing sample $x$ will be classified as the client by the largest similarity. The likelihood function $\operatorname{APM}_{c}(x)$ for class $c$ is a mixture of probabilistic functions which is shown in the following equation:

$$
\operatorname{APM}_{c}(x)=\sum_{j=1}^{5} w_{c, j, t} p_{c, j}(x)
$$

where $p_{c, j}(x)$ for $j=1,2, \ldots, 5$ is defined as the probabilistic function in (11) and $w_{c, j, t}$ is the weighting value which can be expressed in the following equation:

$$
\begin{aligned}
& p_{c, j}(x)=\left(\frac{1}{(2 \pi)^{d / 2}}\right)\left(\frac{1}{\sigma_{c, j, t}{ }^{d}}\right) \\
& \times \exp \left(-\frac{1}{2}\left(x-\mu_{c, j, t}\right)^{T}[\Sigma]^{-1}\left(x-\mu_{c, j, t}\right)\right), \\
& \sum_{j=1}^{5} w_{c, j, 1}=1 .
\end{aligned}
$$

With the assumptions in (13), the probabilistic function (11) can be simplified to (14). And our initial weight $w_{c, j, 1}$ is set

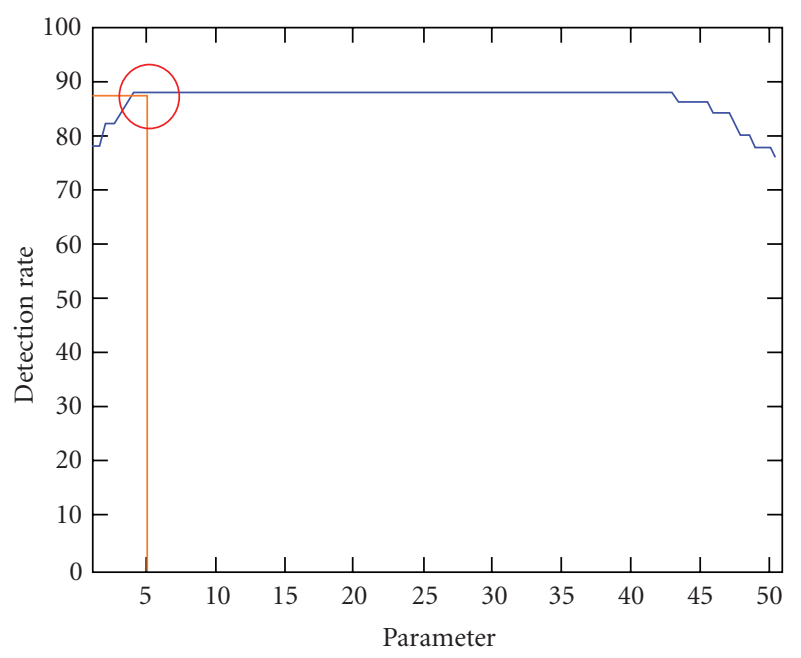

FIGURE 13: The detection rate with respect to different $j$ of the covariance matrix.

to be 0.2 for each $j$-head orientation:

$$
\begin{gathered}
|\Sigma|=\sigma_{c, j, t}^{2 d} \longrightarrow|\Sigma|^{1 / 2}=\sigma_{c, j, t}^{d}, \\
{[\Sigma]=\sigma_{c, j, t}^{2} \cdot I \longrightarrow[\Sigma]^{-1}=\sigma_{c, j, t}^{-2} \cdot I} \\
p_{c, j}(x)=\left(\frac{1}{(2 \pi)^{d / 2}}\right)\left(\frac{1}{\sigma_{c, j, t}{ }^{d}}\right) \exp \left(-\frac{\left(x-\mu_{c, j, t}\right)^{T}\left(x-\mu_{c, j, t}\right)}{2 \sigma_{c, j, t}{ }^{2}}\right) .
\end{gathered}
$$

The other parameters in (10)-(14) such as $t, d, \mu_{c, j, t}, \sigma_{c, j, t}$ represent the time for updating each client's information, the dimension of input vectors, the mean vector, and the covariance matrix, respectively.

3.2. Parameter Tuning and Adaptive Updating. The covariance matrix $\sigma_{c, j, t}$ may affect the performance of APM, so we are inspired to optimize the covariance matrix $\sigma_{c, j, t}$. We have the face database which contains the images of 10 persons in our simulations, and initialize the covariance matrix $\sigma_{c, j, 0}$ by the variance of training data. We can then obtain the updated covariance matrix $\sigma_{c, j, 1}$ by the following equation:

$$
\sigma_{c, j, 1}=\frac{1}{j} \times \sigma_{c, j, 0} .
$$

The detection rate with respect to different $j$ of the covariance matrix $\sigma_{c, j, 1}$ is shown in Figure 13. The detection rate will be obviously improved for $4<j<43$. We can thus choose the parameter $j$ to be 5 and obtain an optimized covariance matrix $\sigma_{c, j, 1}$ in parameter tuning of APM throughout this paper.

The adaptive updating process focuses on the parameter updating of APM. The design of adaptive updating for APM can improve the detection rate of face identification. As the number of updating iterations increases, APM will become more robust and can be simulated to identify the heads in different orientations of our testing clients more accurately. 


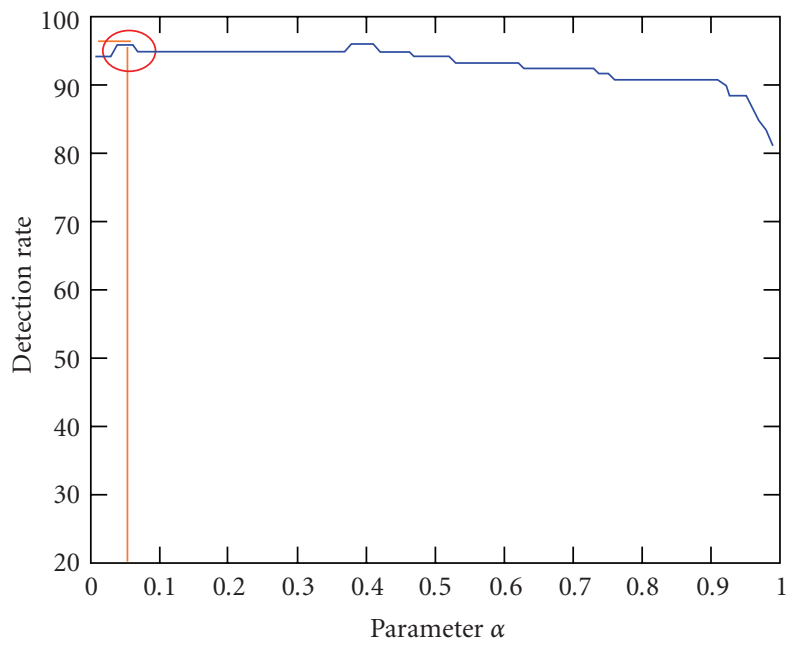

(a)

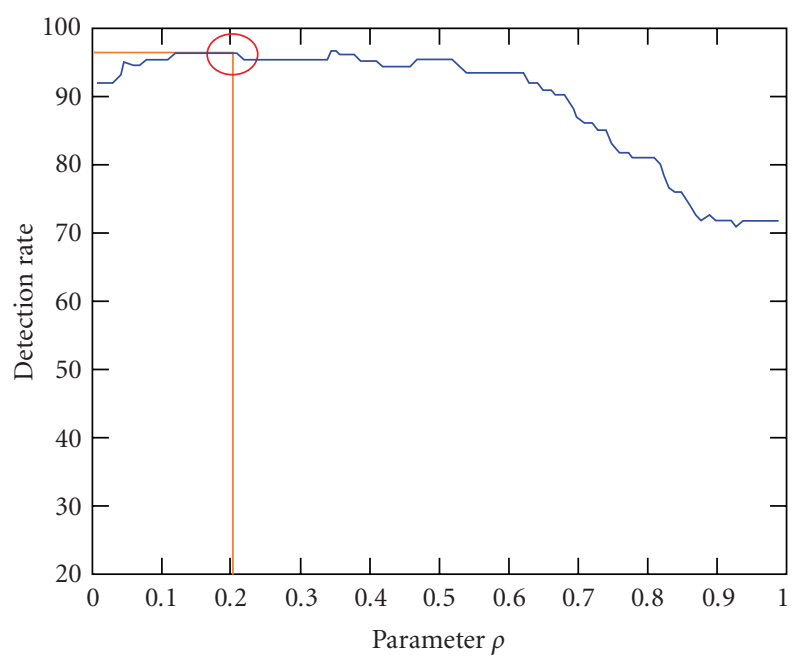

(b)

FIgURE 14: Detection rate, for (a) the parameter $\alpha$ and (b) the parameter $\rho$.
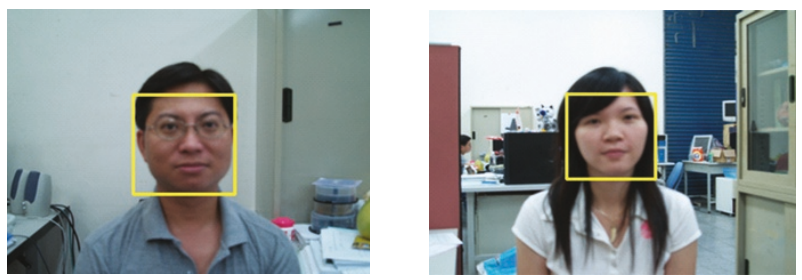

(a)
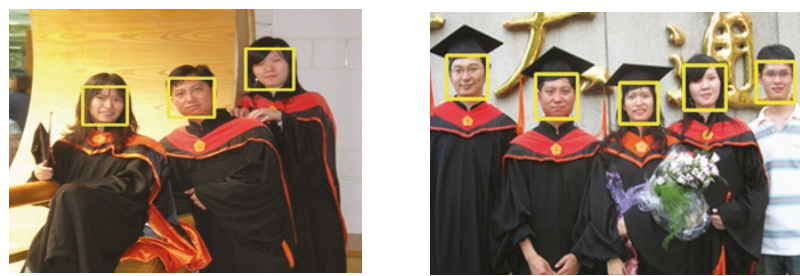

(b)

FIGURE 15: Face detection results for (a) a single face and (b) multifaces in an image.

While a client is identified correctly, the functional APM will be updated immediately by using the following equation:

$$
\begin{gathered}
w_{c, j, t}=(1-\alpha) w_{c, j, t-1}+\alpha\left(M_{c, j, t}\right), \\
M_{c, j, t}= \begin{cases}1, & \text { if mapped, } \\
0, & \text { otherwise, }\end{cases} \\
\mu_{c, j, t}=(1-\rho) \mu_{c, j, t-1}+\rho x \\
\sigma_{c, j, t}^{2}=(1-\rho) \sigma_{c, j, t-1}^{2}+\rho\left(x-\mu_{c, j, t}\right)^{T}\left(x-\mu_{c, j, t}\right),
\end{gathered}
$$

where $\alpha$ and $\rho$ are the learning rates for the weights, mean and covariance matrix. The parameter $\mu_{c, j, t}$ and $\sigma_{c, j, t}$ for unmatched distributions remains the same. The magnitude of learning rates may have influences on the efficiency of APM updating, and a large learning rate will make the likelihood functions of APM over-fitted while the small learning rate results in the worse detection rate. We use the ORL database which contains 40 persons to select the parameter $\alpha$ and $\rho$. Also, we have ten images for each person; five of them are used in the training data, two of them are for the testing data, and the others are for the updating data. Figures 14(a) and 14(b) show the detection rates regarding to the parameter $\alpha$ and $\rho$, respectively. To obtain the best detection rates in the experimental results, we can choose 0.05 for the parameter $\alpha$ and 0.2 for the parameter $\rho$.

\section{Experimental Results}

The experimental results could be divided into two sections, face detection and face identification. We also compared our face detection results with OpenCV (Open Source Computer Vision Library Community). For experiment in face identification, both of face detection and face identification had to work together. Our proposed system could work well for both offline and online testing. Our online testing mechanism could automatically capture images frame by frame from a fixed camera in order to achieve the real-time purposes, and update parameters of APM or add new clients.

4.1. Face Detection. Figures $15(\mathrm{a})$ and $15(\mathrm{~b})$ presented the results of face detection for an image with a single face or multiple faces. In the case of multipersons with different sizes of faces, the proposed face detector system could precisely locate the regions of faces. In order to estimate the performance of the face detector in a more quantized manner, we would show the performance of our system by estimating the detection rates, error rates, and the numbers of false accept images. The testing set consisted of 100 


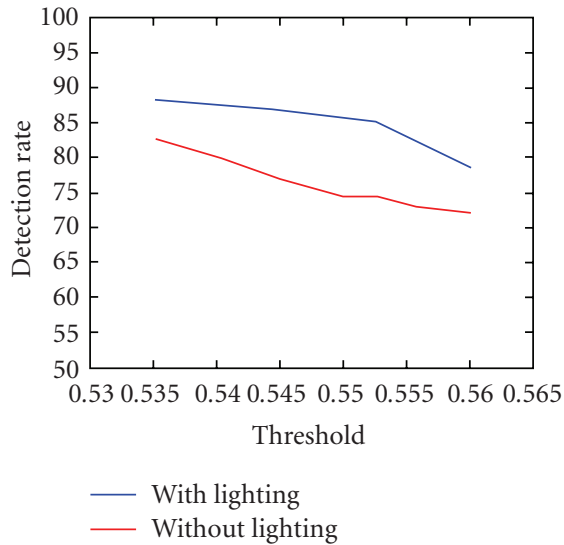

(a)

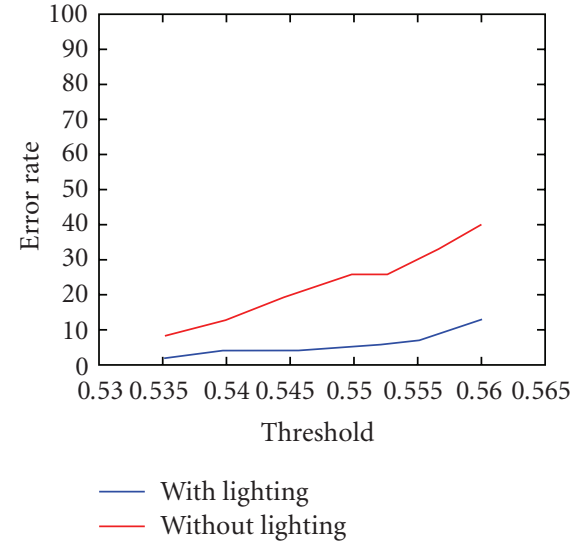

(b)

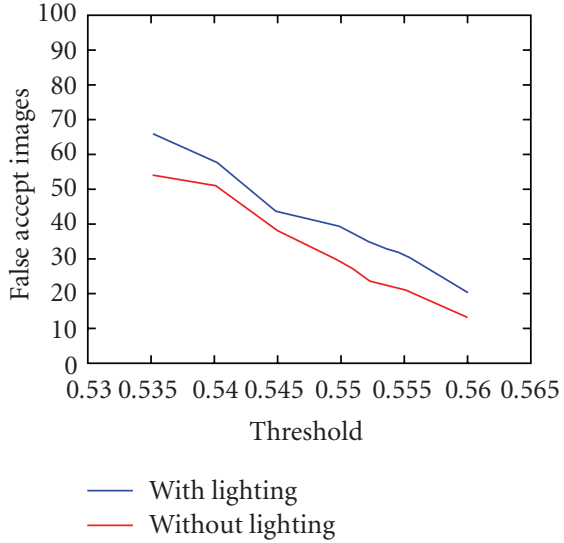

(c)

FIGURE 16: The performance of face detectors in different thresholds for (a) the detection rates, (b) The error rates, (c) The numbers of false accept images.
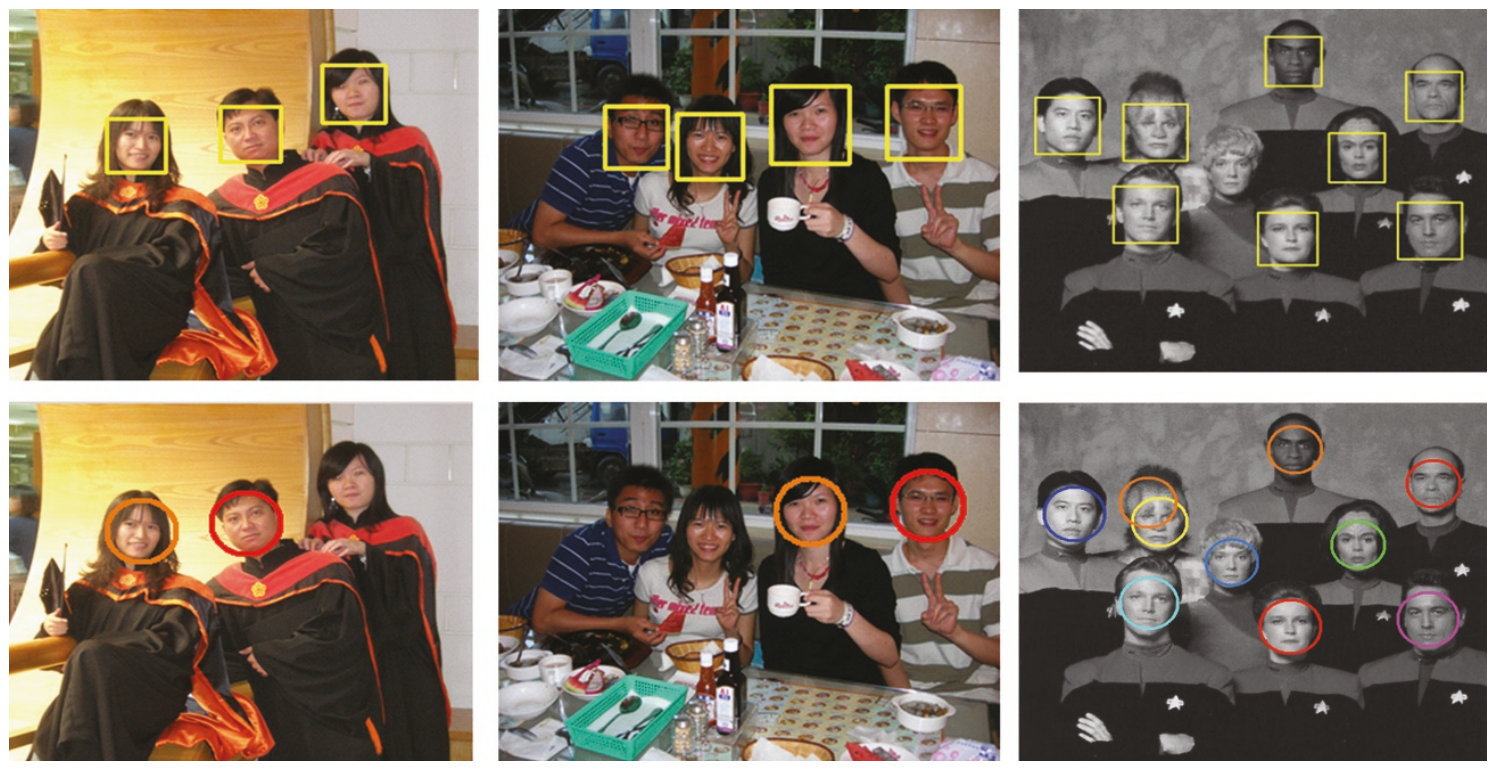

FIGURE 17: Face detection results of the proposed system and OpenCV.

pictures with 434 labeled frontal faces, which were collected randomly from BaoDataBase, Carnegie Mellon Test Images, and the collected pictures of our own. Table 1 displayed the comparison results of face detectors between with and without lighting normalization. Figures 16(a)-16(c) showed the detection rates, error rates, and the numbers of false accept images in different thresholds. We tested 7 different thresholds for our face detector, and calculated the accuracy, error, and the numbers of false accept images correspondingly. In our results, the performance of face detectors with lighting normalization would be better than that without lighting normalization. We also compared our face detection system with OpenCV for the same testing set. The testing results of OpenCV were the detection rate $81.36 \%$, error rate $26 \%$, and the number of false-accept images 5 . Although the number of false-accept images by OpenCV's was smaller than that by our method in some special case, the detection rate by our method would be better than that by OpenCV's for the threshold value smaller than 0.56. Figure 17 demonstrated the results of face detection where the images in the top and bottom row images indicated the detected results of our proposed face detector system and OpenCV's, respectively. It could be observed that our face detector could localize most of face regions precisely except the images shown in the second row. Most of the erroneous results occurred because the face images in the used database were most from Westerns but not from Asians.

4.2. Face Identification. Table 2 illustrated the comparisons of the proposed face identification method PCA+APM and 
TABLE 1: The comparisons of face detectors between with and without lighting normalization.

\begin{tabular}{lcccccc}
\hline \multirow{2}{*}{ Threshold } & \multicolumn{2}{c}{ Detected rate (\%) } & \multicolumn{2}{c}{ Error rate (\%) } & \multicolumn{2}{c}{$\begin{array}{c}\text { Numbers of false accept images } \\
\text { without }\end{array}$} \\
\hline 0.5600 & 72.02 & with & without & with & 13 & 20 \\
0.5550 & 73.12 & 78.60 & 40 & 7 & 21 & 31 \\
0.5525 & 74.53 & 82.80 & 30 & 6 & 23 & 34 \\
0.5500 & 74.55 & 85.11 & 26 & 5 & 29 & 39 \\
0.5450 & 76.92 & 85.57 & 26 & 4 & 38 & 43 \\
0.5400 & 80.24 & 86.70 & 20 & 4 & 51 & 58 \\
0.5350 & 82.86 & 87.29 & 13 & 2 & 54 & 66 \\
\hline
\end{tabular}

TABLE 2: The comparison of characteristics for the proposed method and others.

\begin{tabular}{lccccc}
\hline \multicolumn{2}{c}{ Comparison parameters } & Eigenfaces [17] & PCA+CN [19] & SOM+CN [20] & PCA+APM \\
\hline \multirow{2}{*}{ Method } & Feature & Eigenfaces & Eigenfaces & Self-organizing map & Eigenfaces \\
& Classification & K-nearest neighbor & Neural Network & Neural Network & Adaptive Probabilistic Model \\
\hline \multirow{4}{*}{ Characteristic } & Training time & Quick & Slow & Slow & Quick \\
& Update ability & No & No & No & Yes \\
& Add client & Yes & No & No & Yes \\
& Practicability & Better & Worse & Worse & Better \\
\hline
\end{tabular}

other methods. The compared methods included Eigenfaces, $\mathrm{PCA}+\mathrm{CN}$ (Principal Component Analysis and Convolutional Neural Network), and SOM+CN (Self Organizing Map and Convolutional Neural Network). Our arguments in comparisons were based on the training computational time, the updating ability, and the ability to add the client's database in practicability. Because our system was developed under the considerations of efficiency and performances in real-time environments, the practicability would be the most important one of all the factors. Our system could register new clients and update the client's information online in the real-time cases to achieve practicability.

4.2.1. Offline Testing. Table 3 presented the comparisons of detection rates, in which the testing set used ORL database including 40 persons with ten images of each. We selected 3 -images, 4-images, and 5-images of each person to be the training data, and the remained for testing. Table 3 showed that the more images of each person used in the training process led to the higher detection rates. The lighting normalization also had a great impact on the detection rate. The detection rate without using lighting normalization was slightly below $\mathrm{SOM}+\mathrm{CN}$ and the methods with lighting normalization, and the detection rate would be higher by about $0.3 \%-3 \%$. In order to test the tolerable degrees of our system, we designed an experiment to measure the accuracy for different numbers of clients in particular. Figure 18 indicated the performance of face identifier with different numbers of clients. The detection rate would achieve 100\% for the number of clients lower than 10 , and decrease when the number of clients exceeded 10 . The detection rate would be reduced to $86 \%$ until the number of clients achieved 63 . It

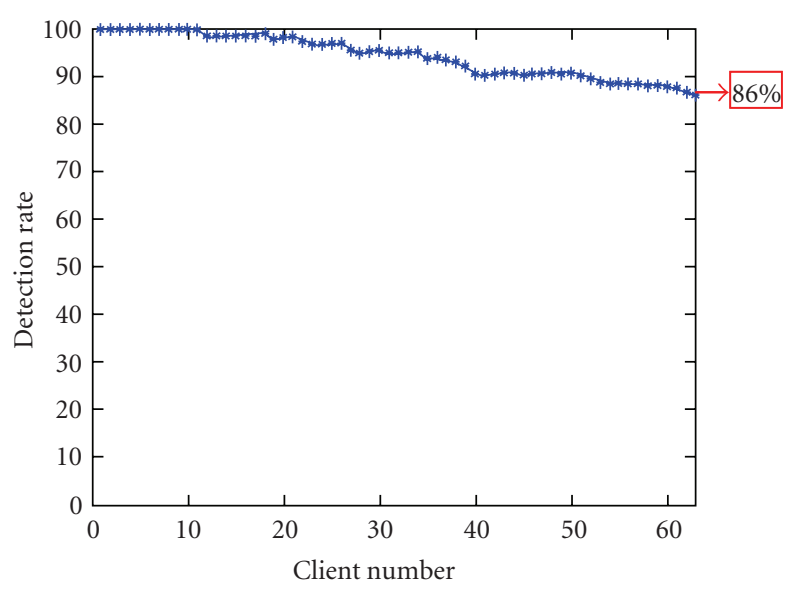

FIGURE 18: The performance of face identifier with respect to the number of clients.

could be acceptable if the detection rate of face identifier was still higher than $80 \%$. In such cases, our system would still be in a tolerable range, and our proposed approach could accept more than 63 clients. For measuring the performances of the adaptive updating process, we used in the ORL database five images of each person for the training data, three for testing, and two for updating. We used the cross validation to estimate the performances of adaptive updating. The upperand lower- lines in Figure 19 illustrated the detection rates after and before the adaptive updating process, respectively. From those experimental data, the performance of face identifier with the adaptive updating process has been obviously improved. 
TABLE 3: The comparison of detection rates of the proposed method and others.

\begin{tabular}{lccccc}
\hline & & \multirow{2}{*}{ Eigenfaces } & PCA+CN & SOM+CN & \multicolumn{2}{c}{ PCA+APM } \\
& & & & without & 86.8 \\
Training images for each person & 3 & 81.8 & 86.8 & 88.2 & 89.3 \\
& 4 & 84.6 & 87.9 & 92.9 & 92.5 \\
& 5 & 89.5 & 92.5 & 96.2 & 92.9 \\
\hline
\end{tabular}

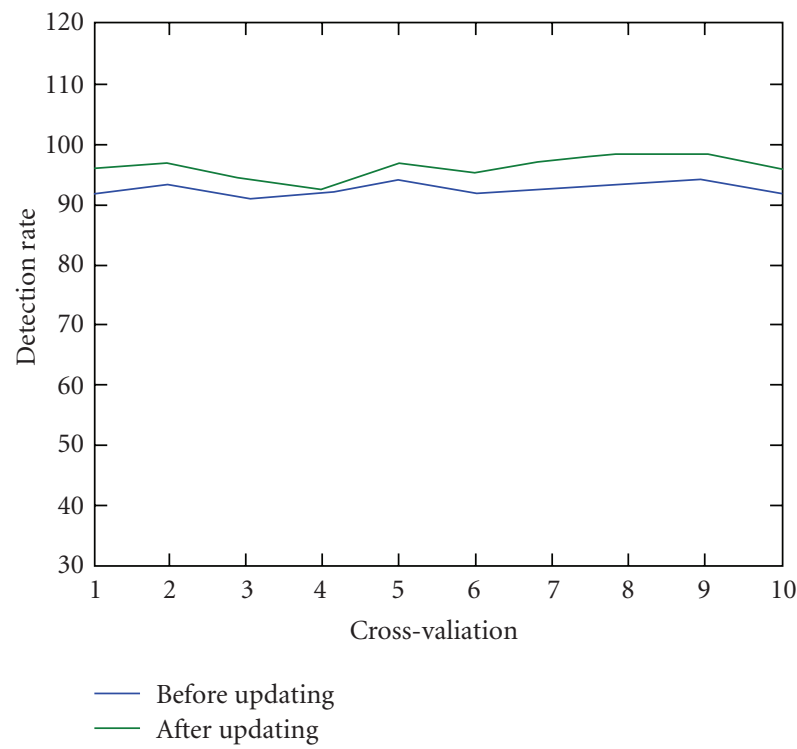

FIGURE 19: The performance of adaptive updating.

TABLE 4: The summarized threshold for distinguishing the clients and impostors.

\begin{tabular}{lcc}
\hline Threshold & FRR & FAR \\
\hline 0 & 4.5 & 89.9 \\
0.000125 & 18.5 & 18.5 \\
0.001 & 32.8 & 5.1 \\
0.002 & 40.5 & 1.3 \\
0.003 & 48.9 & 0 \\
0.004 & 50.7 & 0 \\
\hline
\end{tabular}

We had the total of 26 persons to find the threshold for telling clients from imposters in the proposed system. For 13 out of all clients, five images for each would be used in the training data and the remained were taken for the testing data. We additionally selected five images from the remained 13 impostors to be the testing images. Figure 20 presented the results of selected thresholds for telling the clients from impostors and the tested results would be summarized in Table 4 for some threshold values. The values of thresholds indicated the similarity measures of APM. In Figure 20, FRR/ FAR represented the false reject/accept rate. We made use of the intersection of FAR and FRR to determine the similarity threshold. Based on the experimental result, the value of

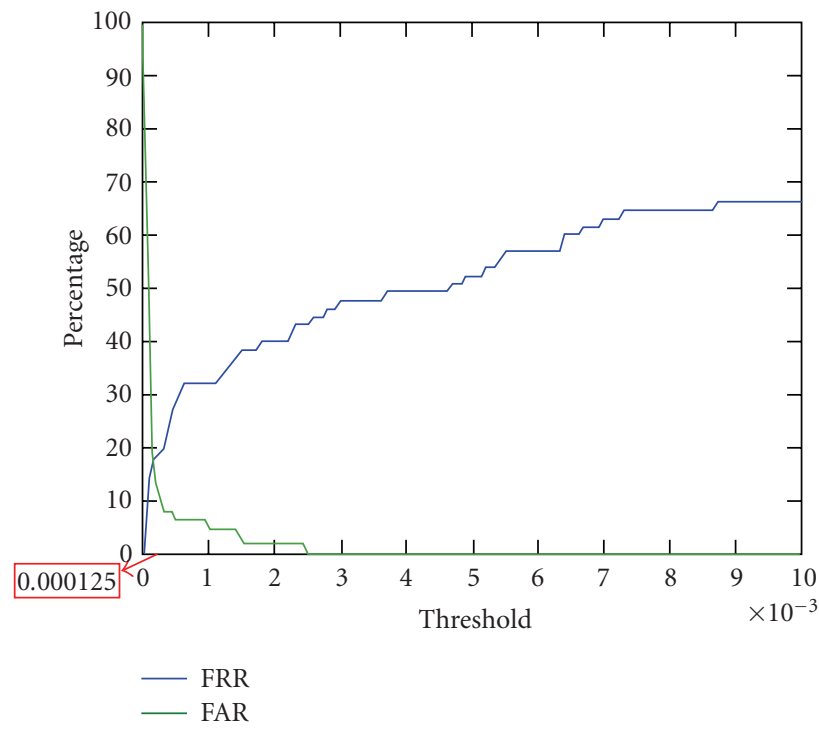

FIGURE 20: The threshold distinguishing the clients and impostors.

threshold for telling the clients from impostors could be set to be 0.000125 .

4.2.2. Online Testing. The experimental results in the online testing were obtained by using the system with the Intel P4 2.60 GHz CPU and 1 GB RAM. The development tool was Borland $\mathrm{C}++$ Builder 6.0 on Window XP OS. The input images were captured from cameras in the $320 \times 240$ resolution. Figure 21 showed some examples of clients in our database. The process to identify a new coming face as a client or an impostor started with capturing an image and extracting the face detector to localize the face regions. The face identifier module then would recognize the faces as the registered clients or impostors. Some results were shown in Figure 22. The registering process for new clients required the images with five different head orientations including the upward, downward, leftward, rightward, and frontal faces. Figure 23 showed the processes in the database when the client was updated. The total run time during the whole process which begins with capturing an image, continues with extraction of face detectors and localizing face regions, and terminates at face recognition and client identification is estimated to be $0.4-0.5$ miniseconds. Moreover, each subprocess such like image acquirement, extraction of face detectors, and client identification accounts for between a 


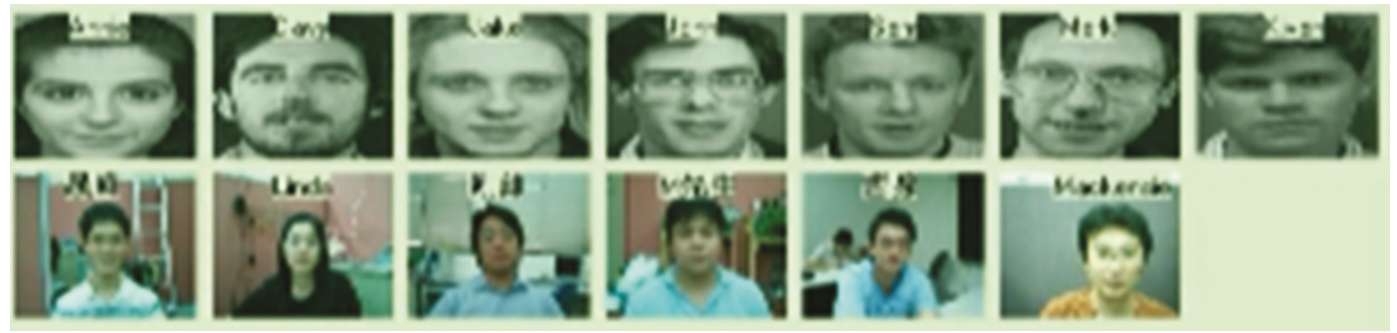

Figure 21: Examples of clients in the database.
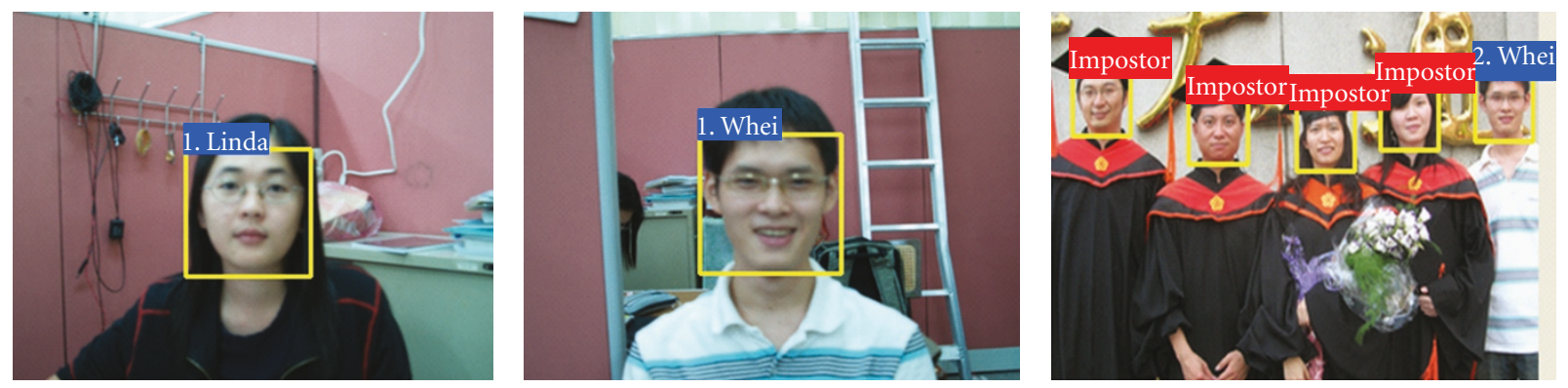

FIGURE 22: The testing results generated from the proposed face identifier.
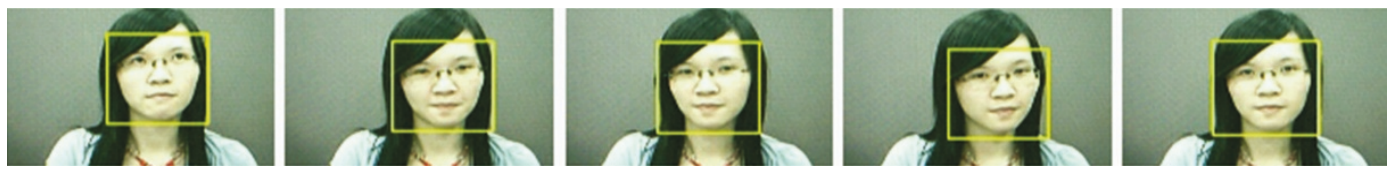

(a)

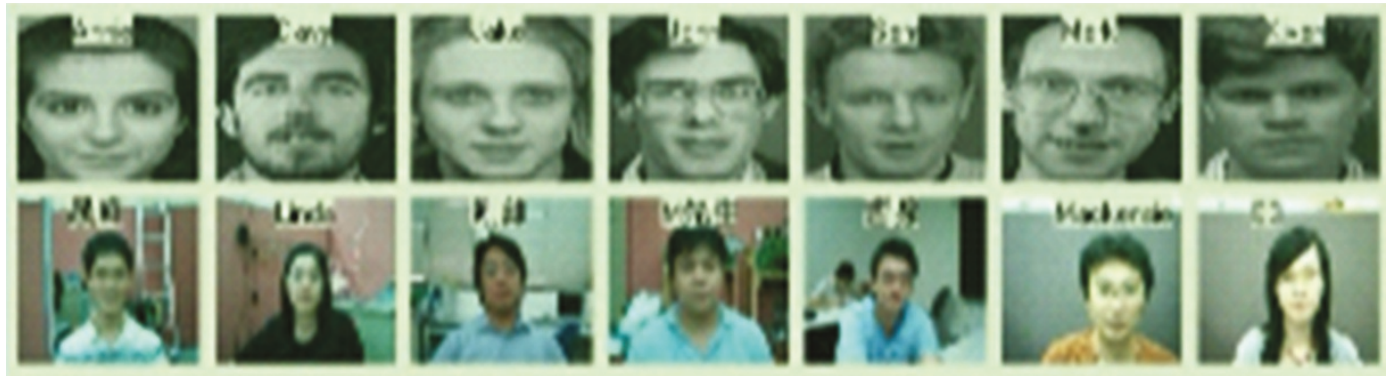

(b)
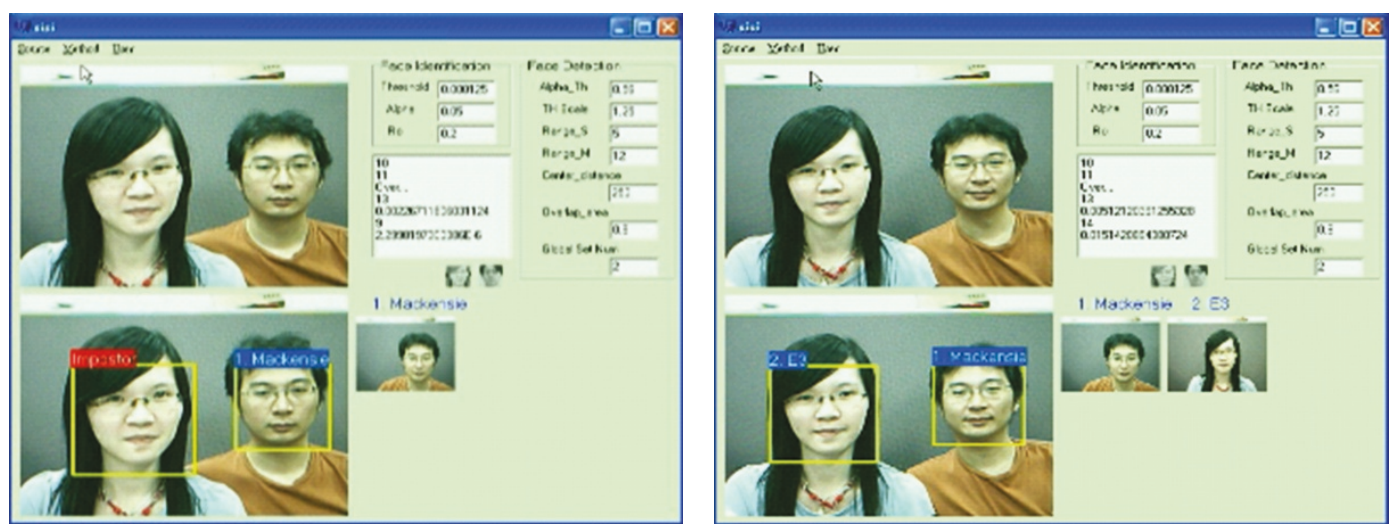

(c)

Figure 23: (a) A new client with different head orientations, (b) An overview of the updating database, (c) Face identifier before and after registering a new client. 


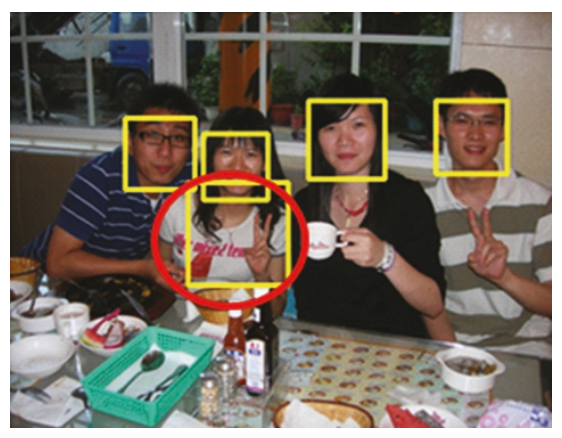

(a)

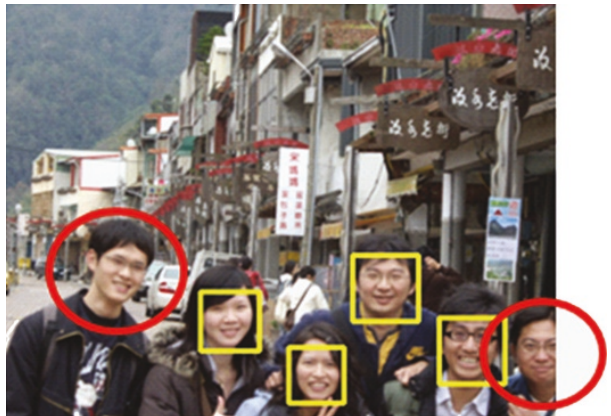

(b)

FIgURE 24: Examples of detection failures in (a) false detection, and (b) missed face regions.

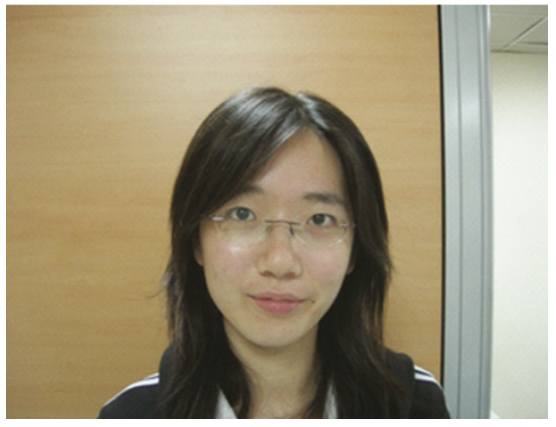

(a)

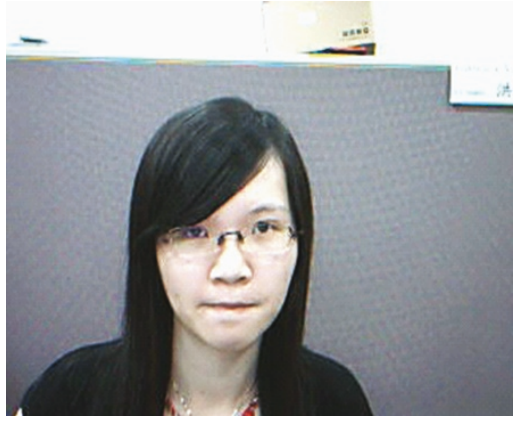

(b)

Figure 25: The example of false acceptance for (a) an impostor and (b) a client.

fourth to a half of the total runtime in a complete process of multiclient detection and identification.

\section{Discussions and Conclusions}

The integration of face detection and face identification for real-time face recognition application has been proposed in this paper. The design of this system focuses on robustness and practicability. We demonstrate our proposed approach to accurately detect the face regions in an image. Besides, the system provides an identification mechanism to identify whom the extracted face of clients belongs to in the database, and a judgment way to regard the detected client as an impostor or a new client. In the face detection, the lighting normalization can actually improve the detection rate and a region-based clustering method is able to deal with the problems of multiple candidates around our target face. However, some nonface images with face-like shapes as shown in Figure 24(a), or partially occluded faces in an image as shown in Figure 24(b) may result in the detection errors. For some special case as shown in Figure 25 where the two clients are too similar to be distinguished from, the false acceptance may occur inevitably. For face identification, an adaptive probabilistic model (APM) is introduced to model the characteristics of clients. According to the design of APM, the system can register a new client and update the information of clients online. By the process of adaptive updating, the weights for different poses and the matched probabilistic functions are adjusted to satisfy the latest information of registered clients. The experimental finally results show that the proposed APM-based technique indeed has a good performance for both the face detection and identification in most cases. And we will consider more exceptional cases which may not be processed by our proposed system in the near future.

\section{Acknowledgments}

This work was supported in part by the Aiming for the Top University Plan of National Chiao Tung University, the Ministry of Education, Taiwan, under Contract 99W962, and supported in part by the National Science Council, Taiwan, under Contracts NSC 99-3114-E-009-167 and NSC 98-2221E-009-167.

\section{References}

[1] A. K. Jain, R. Bolle, and S. Pankanti, Biometrics: Personal Identification in Networked Society, Kluwer Academic Publishers, Dordrecht, The Netherlands, 1999.

[2] D. Zhang, Automated Biometrics: Technologies Systems, Kluwer Academic Publishers, Dordrecht, The Netherlands, 2000. 
[3] C. P. Papageorgiou, M. Oren, and T. Poggio, "A general framework for object detection," in Proceedings of the 6th IEEE International Conference on Computer Vision, pp. 555-562, January 1998.

[4] S. Z. Li, Q. Zhang, H. Shum, and H. J. Zhang, "FloatBoost learning for classification," in Proceedings of the Neural Information Processing Systems, December 2002.

[5] R. Lienhart and J. Maydt, "An extended set of Haar-like features for rapid object detection," in Proceedings of the International Conference on Image Processing (ICIP '02), vol. 1, pp. 900-903, September 2002.

[6] P. Viola and M. J. Jones, "Robust real-time face detection," International Journal of Computer Vision, vol. 57, no. 2, pp. 137-154, 2004.

[7] Y. Freund and R. E. Schapire, "A decision-theoretic generalization of on-line learning and an application to boosting," in Proceedings of the 2nd European Conference on Computational Learning Theory (EuroCOLT '95), pp. 23-57, Springer, 1995.

[8] P. N. Belhumeur, J. P. Hespanha, and D. J. Kriegman, "Eigenfaces vs. fisherfaces: recognition using class specific linear projection," IEEE Transactions on Pattern Analysis and Machine Intelligence, vol. 19, no. 7, pp. 711-720, 1997.

[9] T. F. Cooles, G. J. Edwards, and C. J. Taylor, "Active appearance models," IEEE Transactions on Pattern Analysis and Machine Intelligence, vol. 23, no. 6, pp. 681-685, 2001.

[10] L. Wiskott, J.-M. Fellous, N. Krüger, and C. D. Von Malsburg, "Face recognition by elastic bunch graph matching," IEEE Transactions on Pattern Analysis and Machine Intelligence, vol. 19, no. 7, pp. 775-779, 1997.

[11] K. W. Bowyer, K. Chang, and P. Flynn, "A survey of approaches and challenges in $3 \mathrm{D}$ and multi-modal $3 \mathrm{D}+2 \mathrm{D}$ face recognition," Computer Vision and Image Understanding, vol. 101, no. 1, pp. 1-15, 2006.

[12] W. Zhao, R. Chellappa, P. J. Phillips, and A. Rosenfeld, "Face recognition: a literature survey," ACM Computing Surveys, vol. 35, no. 4, pp. 399-458, 2003.

[13] S. Du and R. Ward, "Face recognition under pose variations," Journal of the Franklin Institute, vol. 343, no. 6, pp. 596-613, 2006.

[14] D. J. Beymer, "Face recognition under varying pose," Tech. Rep. 1461, MIT AI Lab, Massachusetts Institute of Technology, Cambridge, Mass, USA, 1993.

[15] M. Kirby and L. Sirovich, "Application of the Karhunen-Loeve procedure for the characterization of human faces," IEEE Transactions on Pattern Analysis and Machine Intelligence, vol. 12, no. 1, pp. 103-108, 1990.

[16] L. Sirovich and M. Kirby, "Low-dimensional procedure for the characterization of human faces," Journal of the Optical Society of America A, vol. 4, no. 3, pp. 519-524, 1987.

[17] M. Turk and A. Pentland, "Eigenfaces for recognition," Journal of Cognitive Neuroscience, vol. 3, no. 1, pp. 71-86, 1991.

[18] A. Sehad, H. Hocini, A. Hadid, M. Djeddi, and S. Ameur, "Face recognition under varying views," in Proceedings of the 1st IEEE International Workshop on Biologically Motivated Computer Vision, pp. 258-267, May 2000.

[19] R. Ebrahimpour, E. Kabir, and M. R. Yousefi, "Teacherdirected learning in view-independent face recognition with mixture of experts using single-view eigenspaces," Journal of the Franklin Institute, vol. 345, no. 2, pp. 87-101, 2008.

[20] S. Lawrence, C. L. Giles, A. C. Tsoi, and A. D. Back, "Face recognition: a convolutional neural-network approach," IEEE Transactions on Neural Networks, vol. 8, no. 1, pp. 98-113, 1997. 